\title{
Active reduction of elastic objects oscillation tasks
}

\author{
Aleksandr Bokhonsky ${ }^{1}$, Vasiliy Golovin ${ }^{1, *}$, and Mikhail Maistrishin ${ }^{1}$ \\ ${ }^{1}$ Sevastopol State University, Polytechnical Institute, 299053 Sevastopol, Russia
}

\begin{abstract}
Application features of optimal control methods for oscillations of an elastic system stipulated by initial disturbance are being analyzed. The ways of the search control simplifying and their practical implementation are being estimated.
\end{abstract}

\section{Introduction}

The search for oscillation of elastic system control is illustrated by a number of simple examples in [1,2]. If, for example, in [1] an optimal regulator is found by means of analytical construction when the minimum of the quadratic optimality criteria is reached, then in [2] the attention is paid to the method of moments in the search control of elastic system oscillation, it is shown that the power optimal control bears a resonant character.

Works [3-10] are dedicated to the search of optimal controls for the portable motion of elastically deformable systems with a finite and infinite number of degrees of freedom. The construction of control was carried out with the use of the reversible principle of optimality (RPO), the use of which led to appearance of wide class of skew-symmetric controls, ensuring at the minimum possible time, determined from moment proportions, the achievement at the end of the portable motion of absolute or relative quiescence, with some energy savings in practical implementation of controls [10].

However, the skew-symmetric controls proposed and studied in [3-4, 8-9] are not now applicable for suppression of oscillations due to disturbance, for example, in the form of initial conditions (in the absence of a portable motion).

In [11], an attempt was made to search for control of the oscillations of elastic systems (their absorbation) without the use of the algorithm of regulators analytic design, which is related to the need of solving differential or algebraic Riccati equations.

Purpose of research is to analyze the features of the application of optimal control methods for free elastic systems oscillations with the assessment of possible simplification for their practical implementation.

A simple example of an elastic system with one degree of freedom shows the use of various methods for optimal control synthesis by suppression of oscillations.

\footnotetext{
*Corresponding author: vig220@mail.ru
} 


\section{Regulator analytic design}

Oscillations control of an elastic system with one degree of freedom is searched [1]. Equation of controlled motion without taking into account linear-viscous resistance:

$$
\frac{d^{2} x}{d t^{2}}+k^{2} x=\mathrm{U}(t)
$$

where $k$ - natural oscillation frequency. Control of $\mathrm{U}(t)$ is minimizing the quadratic optimality criterion

$$
J=\int_{0}^{T}\left(x^{2}+r \mathrm{U}^{2}\right) d t,
$$

where, if $r=1$, system of algebraic Riccati equations

$$
1-2 k^{2} p_{12}-p_{12}^{2}=0, \quad 2 p_{11}-p_{12} p_{22}=0, \quad 2 p_{12}-2 p_{22}-p_{22}^{2}=0,
$$

solution of equation:

$$
p_{11}=-0,01274 ; \quad p_{12}=-0,01266 ; \quad p_{22}=-2,01258 \text {. }
$$

The optimal regulator in the negative feedback form is described by the correlation $U(t)=-p_{12} x-p_{22} \dot{x}$.

For a frequency of free oscillations $k=2 \pi \mathrm{s}^{-1}$, with initial conditions $x(0)=0,01 \mathrm{~m}$, $\dot{x}(0)=1 \mathrm{~m} / \mathrm{s}$ movement and speed diagrams are reflected on fig. 1,2 .

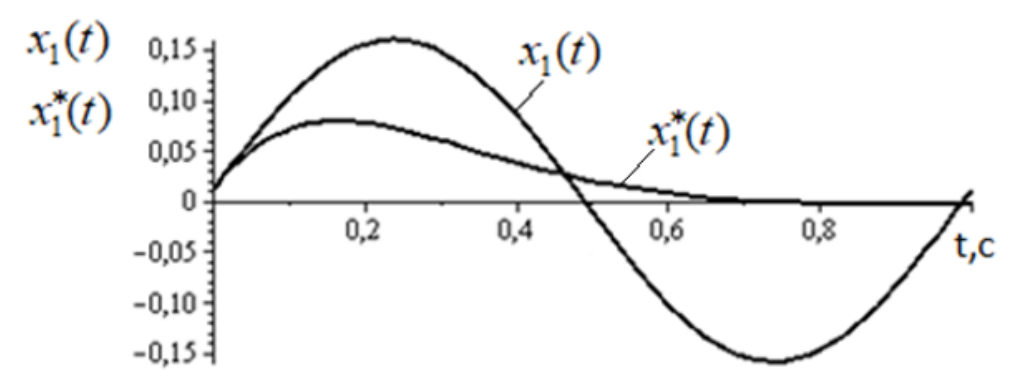

Fig. 1. Movement diagrams: without control, with control.

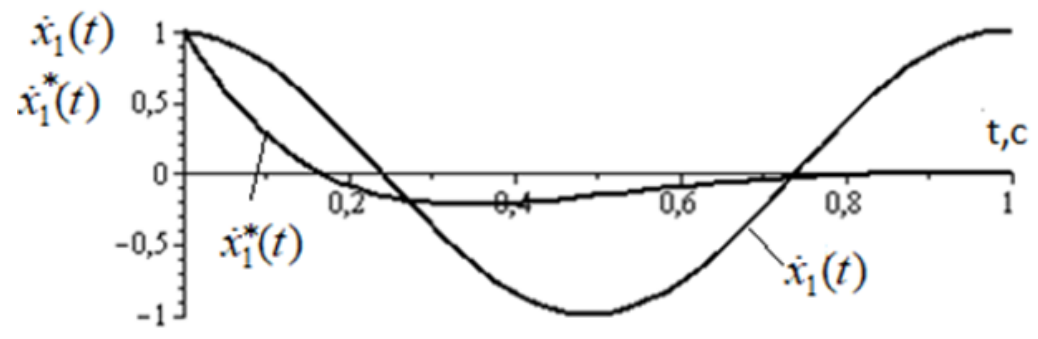

Fig. 2. Speed diagrams: $\dot{x}_{1}(t)$-without control, $\dot{x}_{1}^{*}(t)$-with control. 
Taking into account determined $\mathrm{U}(t)$, equation (1) should be written as follows:

$$
\ddot{x}+k^{2} x=-p_{12} x-p_{22} \dot{x} \quad \text { or } \quad \ddot{x}+p_{2} \dot{x}+\left(k^{2}+p_{12}\right) x=0 .
$$

Feedback, changing the dynamic properties of the original system, amplifies the deformation of the oscillations caused by the initial conditions.

Naturally, to increase the effectiveness of damping, it is not excluded compulsory increase of the gain $p_{22}$ without losing the stability of the system.

\section{Resonance control}

Resonance control example is illustrated in [2]. Resonance control for equation (1) is as follows:

$$
\mathrm{U}(t)=-\frac{1}{\pi}\left(k^{2} q_{1} \sin (k t)+k q_{2} \cos (k t)\right)
$$

where $x(0)=q_{2}, \dot{x}(0)=q_{1}$. Equation's (1) solution, taking into consideration (6)

$$
x(t)=-\frac{1}{2 \pi k}\left(\left(2 q_{2} \pi+q_{1} k\right) \sin k t+\left(2 q_{1} k+q_{2}\right) \cos k t+\left(-q_{2}-q_{1} k^{2} t\right) \cos (k t)-q_{2} k t \sin k t .\right.
$$

Diagram $x(t)$ and $v(t)=\frac{d x}{d t}$ with $k=2 \pi \mathrm{s}^{-1}, q_{1}=0,01 \mathrm{M}, q_{2}=1 \mathrm{~m} / \mathrm{s}$ are reflected on figure 3, showing that in the moment of $t=T=1 \mathrm{~s}$ the quiescent state occurs. Moment ratios $x(T)=0, \dot{x}(T)=0$, as the system of transcendental equations, have one of the common roots $T=1 \mathrm{~s}$, which is natural for resonance control.

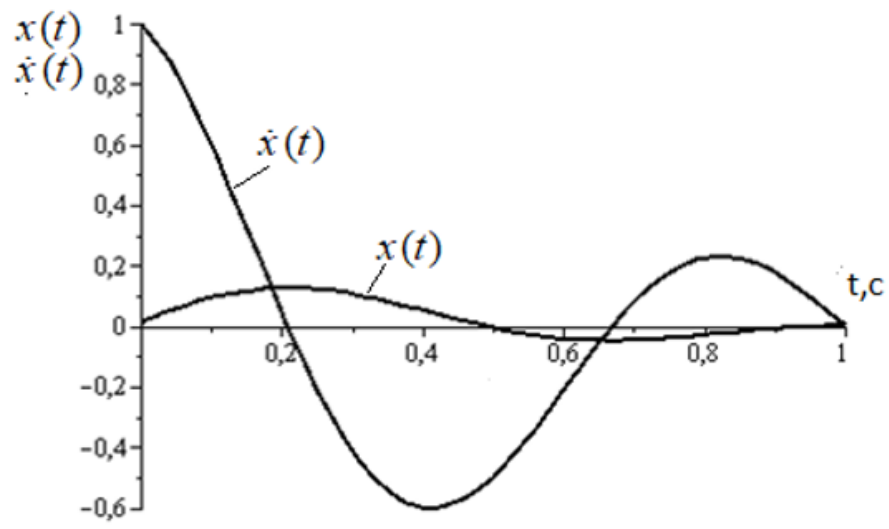

Fig. 3. Diagram $x(t), \dot{x}(t)$ with resonance control.

\section{Simplified algorithm of resonance control searching}

There is a possibility for some simplification of control synthesis algorithm. In this case the right part of equation (1) will be reflected as 


$$
\mathrm{U}(t)=A \cos k t+B \sin k t
$$

Initial condition $x(0)=q_{1}, \dot{x}(0)=q_{2}$. Equation solution (1)

$$
x(t)=\frac{1}{2 k^{2}}\left[\left(2 q_{2} k+B\right) \sin k t-\left(-2 q_{1} k^{2}+A\right) \cos k t+(A-B k t) \cos k t+\sin k t \cdot A k t\right],
$$

where if $T=1 \mathrm{~s}$, then: $A=-2 q_{2}, B=4 \pi q_{1}$.

Diagrams $x(t)$ and $\dot{x}(t)=v(t)=\frac{d x}{d t}$ are reflected on figure 4. Same as in previous case, complete oscillations suppression occurs (when $t=T=1 \mathrm{~s}$ ).

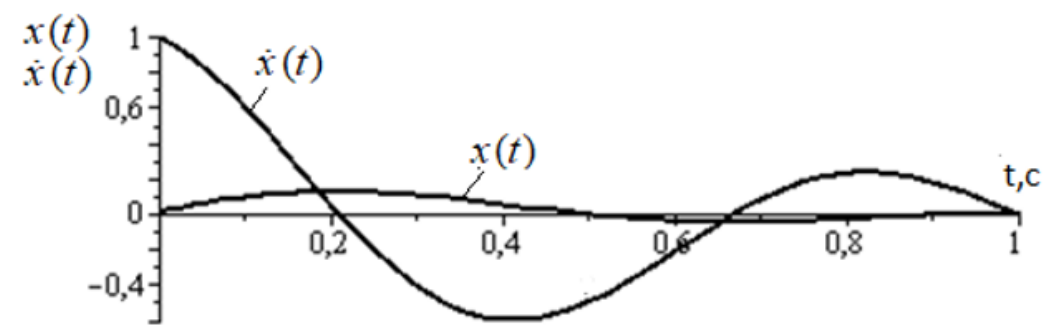

Fig. 4. Oscillations $x(t), \dot{x}(t)$ when simplified resonance algorithm is used.

\section{Nonresonance control}

For nonresonance control it is accepted

$$
\mathrm{U}(t)=A \sin p t+B \cos p t
$$

where $p \neq k$, as before, it is necessary to find such $A$ and $B$, that at fixed moment of time $t=T$ will ensure complete oscillations suppression, so the following conditions are fulfilled: $x(T)=0, \dot{x}(T)=0$.

Further it is expected that $p=n k, n=1,2,3, \ldots, T=\frac{\pi}{p}=\frac{\pi}{n k}$.

Equation solution (1) with the right part (9) satisfying the moment ratio $(x(T)=0$, $\dot{x}(T)=0$ ) leads, after transformation, to the following system of algebraic equations:

$$
\begin{aligned}
& \frac{C_{1}}{k} \sin \left(\frac{\pi}{n}\right)+C_{2} \cos \left(\frac{\pi}{n}\right)+B=0, \\
& -C_{1} \cos \left(\frac{\pi}{n}\right)+k C_{2}\left(\frac{\pi}{n}\right)-A=0,
\end{aligned}
$$

where $C_{1}=q_{2} k^{2}\left(n^{2}-1\right)+A n k, C_{2}=q_{1} k^{2}\left(n^{2}-1\right)+B$.

For this sample it is accepted that $n=2$. System (10) leads to the following: 


$$
A=\frac{3}{4} k q_{2}+\frac{3}{4} k q_{1}, \quad B=-\frac{3}{2} k q_{1}+\frac{3}{2} k q_{2} .
$$

Diagrams $x(t)$ and $v(t)$ with the same initial conditions, reflected on figure 5 , confirm reaching of a quiescent state at time $t=T=1,25 \mathrm{~s}$; thus in this moment of time the control $\mathrm{U}(t)$ has to be disabled.

One of the simplest control model scheme is reflected on figure 6.

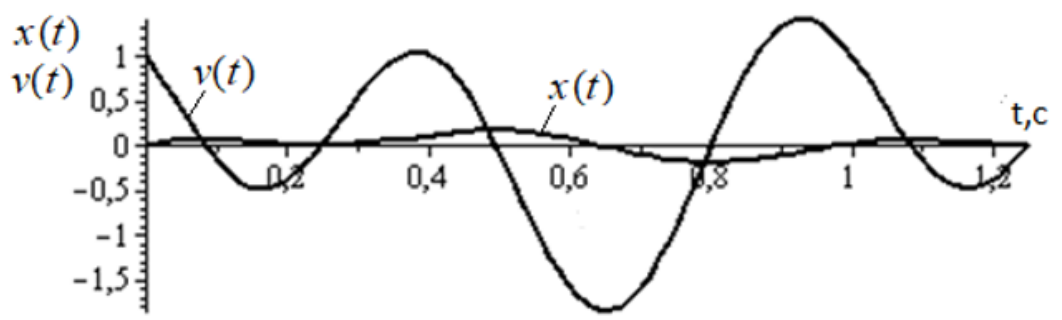

Fig. 5. Diagram of transfer $x(t)$ and speed $v(t)$ in case of non-resonance control.

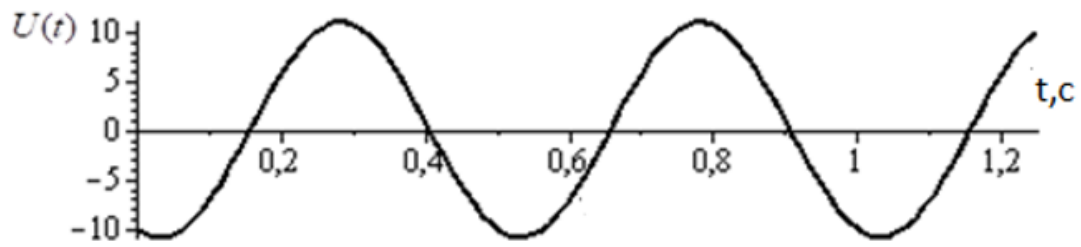

Fig. 6. Non-resonance control diagram.

\section{Unlubricated friction as relay control}

In [8] attention to oscillation suppression with the use of relay control was drawn and it was specified that unlubricated friction can be used as alternative for relay control, thus the right part of equation (1) will be as follows:

$$
\mathrm{U}(t)=-f \cdot \operatorname{sign} \dot{x},
$$

where $f$ - frictional force (per unit weight), which value can be elaborated as a result of consequent approximating with given motion time T. Computational solution of equitation (1) is done in MathCad. 


$$
\begin{aligned}
& \mathrm{x}:=\left(\begin{array}{c}
0.01 \\
1
\end{array}\right) \\
& \begin{aligned}
\mathrm{k} & :=2 \cdot \pi \\
\mathrm{D}(\mathrm{t}, \mathrm{x}) & \left.:=\left[\begin{array}{c}
\mathrm{x}_{1} \\
-\left(\mathrm{k}^{2}\right) \cdot \mathrm{x}_{0}-1.3 \operatorname{sign}\left(\mathrm{x}_{1}\right)
\end{array}\right] \quad \mathrm{Z}:=\operatorname{rkfixed} \mathrm{x}, 0,1.2,12000 \mathrm{D}\right)
\end{aligned}
\end{aligned}
$$

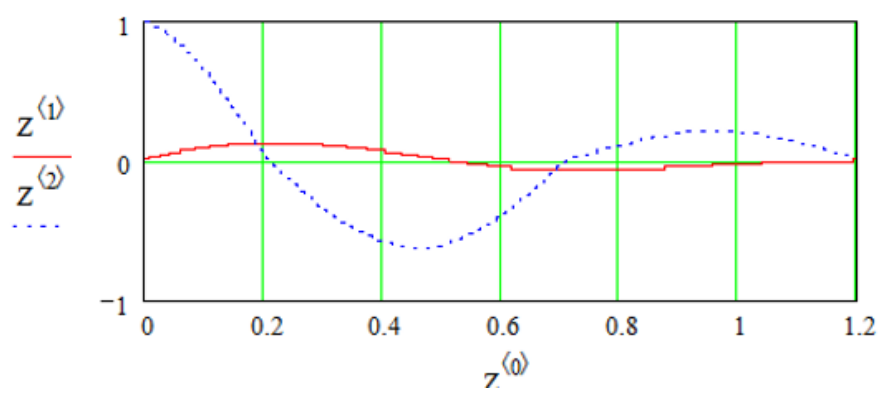

Fig. 7. Unlubricated friction as relay control; diagrams $x(t), \dot{x}(t)$

Diagrams $x(t)$ and $v(t)$, specified on figure 7 , adequately for practice reflect achievement of the controlled motion.

Elementary chart of controls which allows analyzing its mode of behavior in case of change of initial conditions is on figure 8 .

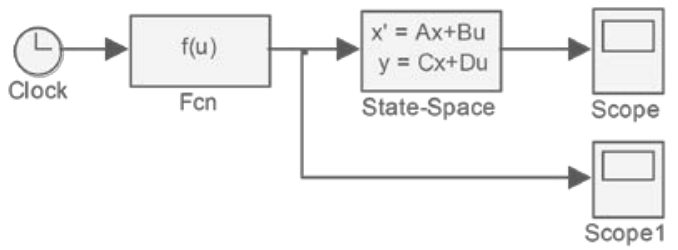

Fig. 8. Flow chart of elastic system model in case of resonant control of oscillations.

\section{Conclusions}

Works [12-15] and others are dedicated to practical implementation of oscillation control of finite rigidity technical objects.

The simplest technical decision in case of complex motion of elastic objects with nonzero initial conditions is use of mixed control: resonant - for suppression of oscillations determined by initial conditions; software - for optimal transition motion to absolute quiescent state.

Still remains relevant search of optimal transition motion of elastic object, which without additional control (in case of non-zero initial conditions) leads at the final stage the system to absolute quiescent state.

Robotechnics, universal mechatronic units development allows implementation in practice of oscillation control of complex elastic systems. The analyzed simplest ways of system oscillation suppression with one degree of freedom covers complex elastic systems with the definite number of degrees of freedom. 


\section{References}

1. V.V. Solodovnikov, Intro to Theory and Elements of the System of Automatic Regulation (Mechanical Engineering, 1985)

2. I.A. Karnovskiy, Y.M. Pochtman, Optimal Control Methods of Deform Systems' Oscillation (Vysha shkola, 1982)

3. A.I. Bohonskiy, N.I. Varminskaya, Variational and Reversible Computations in Mechanics (Sevastopol: SevNTU, 2012)

4. A.I. Bohonskiy, N.I. Varminskaya, M.I. Mozolevskiy, Optimal Control of Deform Objects' Motion: Theory and Mechanical Applications (Sevastopol: SevNTU, 2007)

5. A.I. Bohonskiy, Modeling and analysis of elastic system in motion (Gliwice: Wydawnictwo Politechniki, 2011)

6. A.I. Bohonskiy, N.I. Varminskaya, T.V. Mozolevskiy, Motion Control of the Intended Frequency System. Mechanics, Automatics and Robotechnicsm, №2, pp.7-13 (Novokuzneck: NIC MK, 2018)

7. A.I. Bohonskiy, Modelling and investigation of descents - Continues vibrating mechatronic systems with damping (Wydawichwo Politechniki, Gliwice, 2013)

8. A.I. Bohonskiy, Optimality Reversible Principle (College Textbook: INFRA-M, 2016)

9. A.I. Bohonskiy, Actual Problems of Reversible Computations (Palmarium Academic Publishing, 2017).

10. A.I. Bohonskiy, Energy Intensity of Control Over Object Motion, pp.38-41 (Novokuzneck: NIC MK, 2017).

11. A.I. Bohonskiy, A.V. Isaev, Search for Control Over Oscillations of Elastic Objects in the System of Analytical Calculations, Production processes optimization, 10, pp. 4851 (Sevastopol: Sev NTU, 2007)

12. K.V. Frolov, Oscillations in Engineering, Oscillation and Shock Protection, vol. 6 (M. Mechanical Engineering, 1981)

13. K.V. Frolov, F.A. Furman, Applied Theory of Vibro-protection Systems (Mechanical Engineering, 1980)

14. M.D. Genkin, V.G. Elezov, V.V. Yablonskiy, Methods of Machines Controlled Vibroprotection (M:Science, 1985)

15. M.V. Kolovskiy, Nonlinear Theory of Vibro-protection Systems (M: Science, 1966) 